\title{
Susceptibilidad de las larvas de Aedes aegypti al parasitismo por Romanomermis culicivorax en condiciones de laboratorio y de campo en Oaxaca, México
}

\author{
Alberto Santamarina Mijares, ${ }^{1}$ Rafael Pérez Pacheco ${ }^{2}$ \\ y Sabino Honorio Martínez ${ }^{2}$
}

RESUMEN En junio de 1996, en el Estado de Oaxaca, México, se expusieron larvas de mosquito de la especie Aedes aegypti (Linneo) a los preparásitos infectivos del nemátodo Romanomermis culicivorax, Ross y Smith, en condiciones de laboratorio y de campo. Para los experimentos de laboratorio se utilizaron larvas en estadio I-IV, colectadas en reservorios naturales. Los experimentos se realizaron por triplicado, con 100 larvas de cada estadio larvario por experimento, y se probaron tres dosis de aplicación: 5:1, 10:1 y 15:1 (5, 10 ó 15 preparásitos por larva de mosquito). Para los estudios de campo se tomaron 13 criaderos naturales de A. aegypti con larvas en estadio I-IV y se aplicó una dosis de 15:1 por cada criadero. De acuerdo con los resultados obtenidos en los experimentos de laboratorio, se observó un aumento de la infestación media de las larvas a medida que aumentaba la dosis de aplicación desde 5:1 hasta 15:1. Para la dosis de 10:1, la tasa de parasitismo alcanzó valores de 100,100, 85 y 74\% en las larvas en estadio I, II, III y IV, respectivamente, y, para la dosis de 15:1, valores de 100, 100, 90 y 79\%, respectivamente. Los resultados de las pruebas de campo con la aplicación de una dosis de 15:1 en los 13 reservorios naturales proporcionaron elevadas tasas de parasitismo, con valores entre 80-98\%, lo que demostró la susceptibilidad de esta especie de mosquito al parasitismo por R. culicivorax en el Estado de Oaxaca, México.

Muchos países que padecen enfermedades endémicas transmitidas por insectos vectores, como la malaria y el dengue, están desplegando grandes

\footnotetext{
Instituto de Medicina Tropical "Pedro Kourí", La Habana, Cuba. Toda la correspondencia debe ser enviada a Alberto Santamarina Mijares a la siguiente dirección: Lugareño 105 e/ Luaces y Montoro, Municipio Plaza, CP 10600, Ciudad de La Habana, Cuba.

2 Instituto Politécnico Nacional, Centro Interdisciplinario de Investigación para el Desarrollo Integral Regional (CIIDIR), Oaxaca, Oaxaca, México.
}

esfuerzos por incluir el control de esos vectores entre las actividades de atención primaria de salud, buscando a la vez un incremento de la participación comunitaria. La lucha antivectorial puede ser una herramienta poderosa para controlar las enfermedades transmitidas por vectores, que constituyen una de las principales causas de mortalidad en los países en vías de desarrollo (1).

Aedes aegypti (Linneo) es una de las principales especies de mosquito en el ecosistema urbano. Originario de África, se encuentra distribuido y adaptado a las regiones tropicales y subtropicales del mundo y tiene gran importancia desde el punto de vista médico-epidemiológico, por ser vector de los virus del dengue y de la fiebre amarilla (2). En muchos países de América se pretende alcanzar el control de esta especie, lo cual no es posible sin una sólida estructura de salud y salud ambiental (3). Uno de los principales problemas que enfrenta actualmente la 
lucha contra los insectos vectores, y en particular el control de esta especie, es que el uso excesivo de sustancias químicas, además del riesgo de toxicidad que supone para el hombre y los animales en general, ha llevado a la aparición de resistencia, a la destrucción de los controles naturales del vector, a la contaminación del entorno y al desequilibrio de los ecosistemas. Por estas razones, durante las últimas décadas se ha trabajado en la búsqueda de nuevos métodos de control que sean más asimilables ecológicamente.

Una de las alternativas que está creciendo rápidamente como estrategia de lucha contra los vectores es el uso de agentes biológicos (4). Entre los varios agentes de lucha biológica ensayados, los biolarvicidas basados en preparásitos infectivos del nemátodo Romanomermis culicivorax, Ross y Smith, han pasado las pruebas de inocuidad para su aplicación de campo. Este mermítido es un endoparásito obligatorio que completa su desarrollo en el interior de las larvas de mosquito. En algunas pruebas de laboratorio con larvas de Aedes spp. se han obtenido tasas de infestación entre 98 y 100\% con dosis de 10 preparásitos de $R$. culicivorax por larva (10:1), lo cual sugirió la posibilidad de controlar A. aegypti en condiciones de campo (5). En estudios realizados en las Islas Tokelau para controlar Aedes spp. y A. aegypti, tras la aplicación del preparásito en los huecos de los árboles y en depósitos artificiales se observó una elevada tasa de parasitismo y se confirmó el establecimiento de $R$. culicivorax, que persistió casi tres años tras una única aplicación (6).

El objetivo del presente trabajo consistió en evaluar la susceptibilidad de las larvas de A. aegypti al parasitismo por R. culicivorax en condiciones de laboratorio y de campo en el Estado de Oaxaca, México.

\section{MATERIALES Y MÉTODOS}

\section{Estudio de laboratorio}

El estudio de laboratorio se llevó a cabo en junio de 1996. Los preparásitos utilizados en estos experimentos se ob- tuvieron de cuatro cultivos de nemátodos con 60 días de almacenamiento que fueron inundados con agua destilada ( $\mathrm{pH} 6$ y conductividad de 1,3 $\mu \mathrm{S} / \mathrm{cm})$, con el propósito de inducir la eclosión de los huevos y la emergencia de los preparásitos infectivos. Tres horas después, el agua de los cultivos que contenía los preparásitos infectivos (inóculo) se decantó y colectó en un vaso de precipitación de plástico de $2000 \mathrm{~mL}$ y se determinó su volumen (1 $800 \mathrm{~mL}$ ); mediante el método de dilución volumétrica (7) se calculó que contenía un total de 250000 preparásitos, de los cuales se aplicaron 36000 . Se ensayaron tres dosis de aplicación (5:1, 10:1 y 15:1), con el objetivo de evaluar los efectos del aumento de la dosis sobre la infestación.

Se utilizaron larvas de $A$. aegypti en sus cuatro estadios de desarrollo, obtenidas de diferentes tipos de reservorios, como depósitos de fibra de vidrio y de barro, tanques y cubetas de plástico, localizados todos en los alrededores del Instituto Politécnico del Estado de Oaxaca. De cada reservorio se recolectaron todas las larvas, mediante la decantación del contenido de agua a través de un colector de malla de nilón de $10 \mathrm{~cm}$ de diámetro y $20 \mathrm{~cm}$ de profundidad, con un mango de $1 \mathrm{~m}$ de longitud. Las larvas obtenidas se colocaron en ocho depósitos plásticos de $5000 \mathrm{~mL}$ de capacidad con agua de los propios reservorios y se trasladaron a los laboratorios del Instituto Politécnico. Primero se formaron tres grupos de 1200 larvas, uno por cada dosis de preparásitos $(1: 5,1: 10$ y 1:15), y cada uno de ellos fue dividido en cuatro subgrupos de 300 larvas en estadio I, II, III o IV, respectivamente. A su vez, cada subgrupo de 300 larvas fue dividido en tres grupos de 100, puesto que todos los experimentos se realizaron por triplicado. Los diferentes grupos de larvas se colocaron en charolas plásticas de $46 \times 32 \times 7 \mathrm{~cm}$ con agua de los propios criaderos, con $\mathrm{pH}$ de 5,8, conductividad de $2,4 \mu \mathrm{S} / \mathrm{cm}$ y temperatura de $27{ }^{\circ} \mathrm{C}$ en el momento de la exposición a los preparásitos infectivos.

Al primer grupo de 1200 larvas se le aplicaron 6000 preparásitos, a razón de 500 preparásitos por cada réplica de 100 larvas (dosis de 5:1); al segundo grupo, 12000 preparásitos a razón de 1000 preparásitos por cada réplica de 100 larvas (dosis de 10:1), y al tercer grupo, 18000 preparásitos, a razón de 1500 preparásitos por cada réplica de 100 larvas (dosis de 15:1). Para cada dosis ensayada se colocó un testigo, compuesto por 100 larvas en estadio II, con el objetivo de comparar los resultados obtenidos y determinar la influencia de cualquier otro factor ajeno al sistema de experimentos. Dos días después de la exposición, de cada réplica se tomaron muestras de 40 larvas que fueron disecadas con agujas entomológicas bajo un microscopio estereoscópico para calcular la infestación media (número medio de parásitos por larva) y la tasa de parasitismo (número de larvas parasitadas dividido por el número de larvas examinadas y multiplicado por 100). También se examinó una muestra de 30 pupas provenientes de larvas en estadio IV expuestas a los preparásitos infectivos a dosis de 15:1 para calcular la infestación media.

\section{Estudio de campo}

Para los experimentos de campo, realizados en agosto de 1996, se tomaron 13 reservorios de $A$. aegypti, localizados en los alrededores del Instituto Politécnico del Estado de Oaxaca, consistentes en 10 depósitos de fibra de vidrio con un área de $1 \mathrm{~m}^{2}$ y $60 \mathrm{~cm}$ de profundidad y tres cubetas plásticas de $90 \mathrm{~cm}$ de ancho y $75 \mathrm{~cm}$ de profundidad. Un examen previo a la aplicación del biolarvicida reveló que en el agua de lluvia acumulada en estos reservorios había larvas de $A$. aegypti en estadio I, II, III y IV, con densidades de 180 a 475 larvas $/ \mathrm{m}^{2}$. En los reservorios $1,3,4,7,9,10$ y 11 (grupo I) se observó que alrededor de $90 \%$ de las larvas se encontraban en los estadios I, II y III, y solo $10 \%$, aproximadamente, en el estadio IV. En cambio, en los criaderos 2 , 5, 6, 8, 12 y 13 (grupo II) no se observó dominancia de un estadio sobre otro. Para comparar los resultados obtenidos y detectar la influencia de cualquier otro factor en los experimentos, se colocó un testigo consistente en una 
cubeta de plástico de $1 \mathrm{~m}^{2}$ de superficie y $75 \mathrm{~cm}$ de profundidad, con una densidad de 240 larvas $/ \mathrm{m}^{2}$, de las cuales $95 \%$, aproximadamente, se encontraban en los estadios I, II y III y $5 \%$ en estadio IV. Con el objetivo de determinar su posible influencia sobre la viabilidad de los preparásitos infectivos, se determinaron algunos parámetros físico-químicos, como el $\mathrm{pH}$ y la conductividad del agua, de los reservorios por tratar y del testigo: los valores registrados fueron de 6,2 a 6,4 y 2,1 a 2,8 $\mu \mathrm{S} / \mathrm{cm}$, respectivamente. La temperatura en el momento de las aplicaciones fue de 27 a $28{ }^{\circ} \mathrm{C}$.

Para promover la eclosión de los huevos y la emergencia de los preparásitos infectivos, se inundaron con agua destilada cinco cultivos de nemátodos con 65 días de almacenamiento que contenían huevos de R. culicivorax. Tres horas después se decantó el agua que contenía los preparásitos infectivos y se colectó en un vaso de precipitación plástico de $2000 \mathrm{~mL}$. Se calculó el volumen del inóculo (1 $950 \mathrm{~mL}) \mathrm{y}$, por el método de dilución volumétrica (7), se determinó que contenía un total de 325000 preparásitos. Con una pipeta de $25 \mathrm{~mL}$ se aplicó una dosis de 15 preparásitos por larva de mosquito en cada reservorio. En total se aplicaron 71505 preparásitos infectivos en los 13 reservorios, para lo cual se tuvo en cuenta la densidad larvaria determinada previamente en cada criadero. Dos días después de las aplicaciones se realizó un muestreo en los reservorios tratados y en cada uno se colectaron aleatoriamente 60 larvas que fueron disecadas con agujas entomológicas bajo un microscopio estereoscópico para determinar la infestación media y la tasa de parasitismo. Los cultivos de $R$. culicivorax utilizados en los experimentos tanto de laboratorio como de campo procedían del laboratorio de nemátodos del Instituto de Medicina Tropical "Pedro Kourí" (La Habana, Cuba).

Todos los datos de laboratorio y de campo se procesaron estadísticamente. Previamente se comprobó que los valores de la infestación media no presentaban distribución normal ni homogeneidad de varianza, por lo que se

CUADRO 1. Infestación media y tasa de parasitismo en larvas de Aedes aegypti tras la aplicación de Romanomermis culicivorax a dosis de 5, 10 y 15 preparásitos por larva en condiciones de laboratorio. Oaxaca, México, 1996

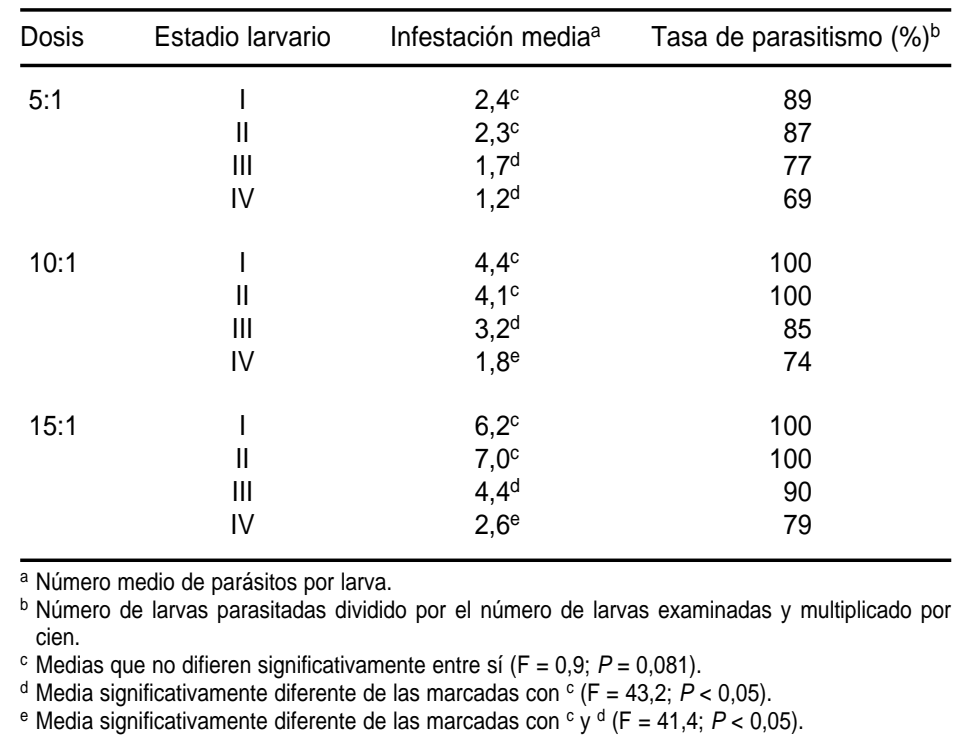

procedió a su transformación logarítmica, con lo que se obtuvo una distribución normal. Además de la infestación media, se registró la tasa de parasitismo, pero no se analizó, pues mostró una correlación cercana a 1 (coeficiente de correlación de Spearman: $\left.\mathrm{r}_{\mathrm{s}}=0,95 ; P<0,001\right)$ con la infestación media. Los datos de laboratorio fueron sometidos a un análisis de varianza (ANOVA) bifactorial para analizar los efectos del estadio larvario y de la dosis de aplicación sobre la infestación media, y los datos de campo a un ANOVA simple para analizar la influencia del estadio larvario sobre la infestación media. Las diferencias entre medias se analizaron mediante la prueba de Duncan, con una probabilidad de error del $5 \%$.

\section{RESULTADOS}

\section{Estudio de laboratorio}

El cuadro 1 muestra los resultados obtenidos en los estudios de laboratorio. Tanto la infestación media como la tasa de parasitismo aumentaron a medida que lo hacía la dosis y, con cualquiera de las tres dosis aplicadas, los mayores valores correspondieron a los estadios más tempranos (I y II), y los menores al estadio IV; el estadio III presentó valores intermedios. Las dosis más elevadas (10:1 y 15:1) proporcionaron tasas de parasitismo de $100 \%$ en las larvas en estadio I y II. El ANOVA bifactorial reveló que tanto la dosis como el estadio larvario influyeron significativamente sobre la infestación media $(\mathrm{F}=125,6 ; P<0,001$ para el efecto de la dosis, y F = 241,2; $P<0,001$ para el efecto del estadio larvario), mientras que la interacción entre dosis y estadio no fue estadísticamente significativa $(\mathrm{F}=1,26 ; P>0,05)$. Se evidenció un incremento de la infestación media a medida que aumentaba la dosis. La prueba de Duncan reveló diferencias significativas entre las infestaciones medias obtenidas con las diferentes dosis $(\mathrm{Q}=8,32 ; P<0,05)$; las medias obtenidas con cada una de las dosis también fueron significativamente diferentes entre los estadios III y IV, y entre cada uno de estos y los estadios I y II $(Q=8,26 ; P<0,05)$, pero no entre estos dos últimos. Con la dosis más elevada (15:1), en los estadios I y II se observó superparasitismo (máximo de 7 nemátodos por larva). El examen de la muestra de 30 pupas provenien- 
CUADRO 2. Infestación media y tasa de parasitismo en larvas de Aedes aegypti tras la aplicación de Romanomermis culicivorax a dosis de 15 preparásitos por larva en condiciones naturales. Oaxaca, México, 1996

\begin{tabular}{lccccc}
\hline Reservorio & DLpre $^{\mathrm{a}}$ & DLpos $^{\mathrm{a}}$ & $\begin{array}{c}\text { Cambio } \\
(\%)\end{array}$ & $\begin{array}{c}\text { Infestación } \\
\text { media }^{\mathrm{c}}\end{array}$ & $\begin{array}{c}\text { Tasa de } \\
\text { parasitismo (\%) }\end{array}$ \\
\hline 1 & 200 & 7 & -97 & $4,3^{\mathrm{e}}$ & 90 \\
2 & 180 & 19 & -89 & $3,0^{\mathrm{f}}$ & 81 \\
3 & 350 & 8 & -98 & $4,1^{\mathrm{e}}$ & 89 \\
4 & 475 & 11 & -98 & $4,2^{\mathrm{e}}$ & 91 \\
5 & 450 & 21 & -95 & $2,8^{\dagger}$ & 80 \\
6 & 380 & 16 & -96 & $3,2^{\mathrm{f}}$ & 83 \\
7 & 426 & 9 & -98 & $4,6^{\mathrm{e}}$ & 96 \\
8 & 415 & 20 & -95 & $3,4^{\dagger}$ & 85 \\
9 & 402 & 6 & -99 & $4,2^{\mathrm{e}}$ & 93 \\
10 & 395 & 8 & -98 & $4,5^{\mathrm{e}}$ & 94 \\
11 & 215 & 12 & -94 & $4,8^{\mathrm{e}}$ & 98 \\
12 & 460 & 23 & -95 & $3,3^{\dagger}$ & 84 \\
13 & 419 & 22 & -95 & $3,0^{\dagger}$ & 82 \\
Control & 240 & 283 & 18 & - & - \\
\hline
\end{tabular}

a DLpre y DLpos: densidad larvaria (número de larvas por $\mathrm{m}^{2}$ ) antes y después de la aplicación de $R$. culicivorax.

${ }^{b}$ Cambio porcentual calculado con la fórmula: [(valor final - valor inicial) / valor inicial] $\times 100$, donde los valores inicial y final son, respectivamente, los registrados antes y dos días después de la aplicación de $R$. culicivorax.

c Número medio de parásitos por larva.

d Número de larvas parasitadas dividido por el número de larvas examinadas y multiplicado por cien.

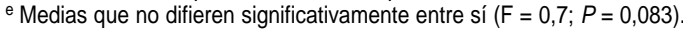

${ }^{\dagger}$ Medias significativamente diferentes de las marcadas $\operatorname{con}^{\mathrm{e}}(\mathrm{F}=44,3 ; P<0,05)$.

tes de larvas en estadio IV infectadas con los preparásitos reveló una infestación media de 2,5 y una tasa de parasitismo de $79 \%$. En los testigos no se observo ningún tipo de alteración; las larvas crecieron normalmente.

\section{Estudio de campo}

Los resultados de la aplicación de R. culicivorax en los 13 reservorios de A. aegypti demostraron la susceptibilidad de esta especie de mosquito al parasitismo por el nemátodo en condiciones de campo. En el cuadro 2 se presentan los resultados obtenidos. El mayor parasitismo ocurrió en los criaderos del grupo I, con infestaciones medias de 4,1 a 4,8 , frente a 2,8 a 3,4 en los reservorios del grupo II. Mediante ANOVA simple se encontraron diferencias significativas $(F=6,43$; $P<0,05)$ entre los valores de la infestación media en los 13 reservorios. La prueba de Duncan reveló que la infestación media fue significativamente diferente en los reservorios de los grupos I y II. Se observó además un mayor parasitismo en los estadios más tempranos (I y II). El examen de las larvas colectadas en cada reservorio $48 \mathrm{~h}$ después de las aplicaciones reveló que la mayoría de las larvas de $A$. aegypti estaban invadidas por el mermítido, con tasas de parasitismo entre 80 y $98 \%$. Las poblaciones de larvas en los 13 reservorios fueron sustancialmente reducidas a consecuencia de la emergencia de los parásitos y la consiguiente muerte de las larvas hospederas (cuadro 2). En los testigos no se observo ningún tipo de alteración; las larvas crecieron normalmente.

Tanto en los experimentos de laboratorio como en los estudios de campo, los parámetros físico-químicos, como el $\mathrm{pH}$, la conductividad y la temperatura del agua no tuvieron efectos adversos detectables sobre las larvas de mosquitos ni tampoco sobre la viabilidad y capacidad infectiva de los preparásitos.

\section{DISCUSIÓN}

Las pruebas de laboratorio realizadas para determinar la susceptibilidad de los diferentes estadios larvarios de
A. aegypti a la infestación por R. culicivorax proporcionaron resultados muy similares a los descritos en estudios anteriores (8-11). Los estadios larvarios I y II fueron los que presentaron la mayor susceptibilidad, muy similar en ambos, seguidos por el estadio III y el IV, que fue el que presentó menor susceptibilidad. El examen de las larvas colectadas 48 horas después de la exposición reveló que muchas de las larvas en estadio I y II estaban superparasitadas, principalmente con la dosis más elevada (15:1), lo cual confirma su mayor susceptibilidad en estos estadios. En general, las disecciones efectuadas permitieron observar los parásitos (habitualmente de $1 \mathrm{~mm}$ de longitud) dentro del hemocele de las larvas de esta especie de mosquito. La infestación media hallada en la muestra de 30 pupas examinada supone que el parasitismo suele transcurrir normalmente a través de la fase de pupa hasta la fase adulta del mosquito: el parásito penetra en la fase de larva y se desarrolla normalmente a través de la fase de pupa y adulto del mosquito, hasta su emergencia en esta última fase.

Al igual que en los experimentos de laboratorio, los experimentos de campo proporcionaron elevados valores de infestación media, especialmente en los estadios larvarios más tempranos. El mayor parasitismo se registró en los reservorios del grupo I, hecho que quizás pueda explicarse por la presencia de poblaciones de larvas más jóvenes, con una cutícula menos espesa (12). Al comparar los 13 reservorios tratados y el reservorio de control se verificó que la dosis de 15:1 proporcionó una importante reducción de los índices de densidad larvaria de A. aegypti (cuadro 2). En el criadero de control se observó un ligero incremento de la densidad larvaria, debido a la puesta y eclosión de nuevos huevos.

Estudios de campo en el oeste de Samoa e Islas Tokelau demostraron la capacidad parasítica de $R$. culicivorax que, cuando se depositó en las axilas de árboles Pandanus sp. a dosis de 1000 a 2000 preparásitos, proporcionó tasas de parasitismo entre 59 y $64 \%$ en 
larvas de Aedes sp. (13). Hay publicados otros resultados positivos sobre la aplicación de mermítidos para el control de las poblaciones larvarias de mosquitos de importancia médico-epidemiológica $(14,15)$, que, al igual que los resultados del presente estudio, indican que el uso de mermítidos podría constituir un método más para alternar con algunos larvicidas químicos como el abate, sobre todo si se tiene en cuenta que un estudio reciente de 102 muestras de A. aegypti procedentes de 16 países, desde Sudamérica hasta las Bahamas, pasando por la cadena de islas del Caribe, evidenció la resistencia manifestada por esta especie tanto al larvicida abate (temephos) como al adulticida malathión, que se han venido utilizando durante los últimos 30 años (16).

Los parámetros físico-químicos, como el $\mathrm{pH}$, la conductividad y la temperatura del agua no tuvieron una influencia negativa sobre la capacidad infectiva de R. culicivorax. La suscepti- bilidad de A. aegypti al parasitismo por $R$. culicivorax, principalmente en sus estadios larvarios más tempranos y en las pruebas tanto de laboratorio como de campo, sugiere que este parásito podría constituir un buen candidato para ser utilizado en el control biológico de $A$. aegypti. La utilización de nemátodos en la lucha contra los mosquitos no está exenta de potenciales inconvenientes, como la necesidad de una previa producción masiva en bioplantas, que a su vez requiere el mantenimiento de un insectario. Además, sus limitaciones en algunos ambientes contaminados podrían hacerlo poco atractivo, pero su uso en la práctica para la lucha contra otras especies de mosquitos, como los anofelinos, los resultados satisfactorios obtenidos, su capacidad de reciclaje y lo económico que resulta su obtención masiva, son aspectos positivos que deberían tenerse en cuenta en el momento de elegir métodos alternativos de lucha contra los mosquitos.
En resumen, los experimentos de laboratorio revelaron que el aumento de la dosis de preparásitos por larva de mosquito desde 5:1 hasta 15:1 incrementó los índices de infestación en larvas; los experimentos de laboratorio y de campo evidenciaron que las larvas en estadios más tempranos resultaron más vulnerables a la invasión por los preparásitos infectivos, y los experimentos de campo demostraron que la dosis de 15:1 resultó altamente eficaz para reducir las densidades de larvas de $A$. aegypti (cuadro 2) en 13 reservorios naturales, lo cual parece indicar que las poblaciones de larvas de mosquito de esta especie del Estado de Oaxaca, México, son susceptibles al parasitismo por el nemátodo R. culicivorax.

Los valores de algunos parámetros físico-químicos, como $\mathrm{pH}$, conductividad y temperatura, calculados en las aguas de los 13 reservorios tratados, aparentemente no interfirieron con la capacidad infectiva de los preparásitos infectivos de R. culicivorax.

\section{REFERENCIAS}

1. Dobrokhotov B. Alternatives to chemical methods for vector control. Ann Soc Belg Med Trop 1991;71(supl. 1):27-33.

2. Varma MGR. Mosquito-borne virus diseases. En: World Health Organization. Geographical distribution of arthropod-borne diseases and their principal vectors. Geneva: WHO; 1989. (Document WHO/VBC/89.967). [http:// dataserver. ciesin.org/docs/001-613/001-613. html]

3. World Health Organization. Report of an informal consultation on the detection, isolation, indentification and ecology of biocontrol agents of disease vectors. Geneva: WHO; 1987. (Document TDR/BVC/GE/873).

4. TDR. Progress Report 1991-92. Geneva: WHO; 1993.

5. Mitchell T, Mitchell C. Susceptibility of Aedes pseudoscutellaris and Aedes polynesiensis to infection by Romanomermis culicivorax in the laboratory. Mosquito News 1982;42:396-399.

6. Laird M, Urdang J, Tinielu I. Establishment and long-term survival of Romanomermis culicivorax in mosquito habitats, Tokelau Islands. Mosquito News 1982;42:86-92.

7. Petersen JJ, Willis OR. Procedures of the mass rearing of a mermithid parasite of mosquitoes. Mosquito News 1972;2(32):226-230.
8. Petersen JJ, Willis OR. Some factors affecting parasitism by mermithid nematodes in southern house mosquito larvae. J Econ Entomol 1970;63:175-178.

9. Petersen JJ. Comparative suceptibility of larval mosquitoes exposed separately by instar or in mixed populations to the nematode Romanomermis culicivorax. J Nematol 1981;13: 228-229.

10. Santamarina MA, González BR. Capacidad de infestación de Romanomermis culicivorax (Ross y Smith 1976) (Rhabditidae: Mermithidae) en larvas de Aedes (S) aegypti (Linnaeus, 1762) (Diptera: Culicidae) en condiciones de laboratorio. Rev Cub Med Trop 1986;38:331-334.

11. Santamarina MA, Pérez PR. Efecto patogénico del nemátodo Romanomermis iyengari (Nematoda: Mermithidae) en larvas de mosquito Aedes aegypti (Diptera: Culicidae) en condiciones de laboratorio en el Estado de Oaxaca, México. Rev Cub Med Trop 1998;50:8-11.

12. Petersen JJ, Willis OR. Experimental release of a mermithid nematode to control Anopheles mosquitoes in Louisiana. Mosquito News 1974;34:316-319.

13. Pillai J. Recent developments concerning the use of nematodes for vector control. Unpublished report to WHO 1980, 16 pages.
14. Santamarina MA, Pérez PR, Honorio STM, Cantón LE, Flores GA. The Romanoermis iyengari parasite for Anopheles pseudopunctipennis suppression in natural habitats in Oaxaca State, Mexico. Pan Am J Public Health 1999;5: 23-28.

15. Santamarina MA, Bellini AC. Producción masiva de Romanomermis iyengari (Nemathoda: Mermithidae) y su aplicación en criaderos de anofelinos en Boa Vista (Roraima), Brasil. Rev Panam Salud Publica 2000:7;155-161.

16. Rawlins SC. Spatial distribution of insecticide resistance in Caribbean populations of Aedes aegypti and its significance. Pan Am J Public Health 1998;4:243-251.

Manuscrito recibido el 17 de noviembre de 1999 y aceptado para publicación, tras revisión, el 10 de julio de 2000. 
ABSTRACT In June 1996 in the state of Oaxaca, Mexico, larvae of the mosquito species Aedes aegypti were exposed to infective preparasites of the nematode Romanomermis culicivorax, Ross and Smith, in the laboratory and in the field. For the laboratory experiments larvae in instars I-IV were used; they had been collected in natural reservoirs. The laboratory experiments were carried out in triplicate, with 100 larvae of each larval stage per experiment. Three preparasite application dosage ratios were tested: 5, 10, or 15 preparasites per mosquito larva. For the field studies $13 \mathrm{~A}$. aegypti outdoor breeding sites were used, with larvae in instars I-IV and a 15:1 preparasite dosage ratio. With the laboratory experiments, an increase was observed in the average infestation of the larvae as the preparasite application ratio was increased from 5:1 to $15: 1$. With a $10: 1$ ratio, the rates of parasitism were $100 \%, 100 \%, 85 \%$, and $74 \%$ in the larvae in instars I, II, III, and IV, respectively; for the 15:1 preparasite ratio, parasitism rates were $100 \%, 100 \%, 90 \%$, and $79 \%$, respectively. The field tests with the $15: 1$ preparasite dosage ratio in the 13 outdoor reservoirs produced parasitism rates of $80 \%$ to $98 \%$, thus demonstrating the susceptibility of this species of mosquito to parasitism by R. culicivorax in Oaxaca, Mexico.

Whatever the several countries may do with regard to the modification of their systems of practice and of service, it seems to me that the scientific study of health and disease in man-the most complex of all social animals-must henceforward concern itself to an ever-increasing degree with the interactions and correlations of disease and health with changing social circumstance.

[Hagan lo que hagan los diferentes países con respecto a la modificación de sus sistemas de práctica y de servicios, me parece que el estudio científico de la salud y la enfermedad en el hombre -el más complejo de todos los animales sociales- debe preocuparse de hoy en adelante cada vez más de las interacciones y correlaciones de la enfermedad y la salud con las circunstancias sociales cambiantes.]

John Ryle, Changing Disciplines, 1948 